\title{
Drone-Aid: An Aerial Medical Assistance
}

\author{
Vaishali Nimilan, Gunaselvi Manohar, R.Sudha, Pearley Stanley
}

\begin{abstract}
Paper Setup must be in A4 size with Margin: Top 0.7”, Bottom 0.7", Left 0.65", 0.65", Gutter 0", and Gutter Position Top. Paper must be in two Columns after Authors Name with Width 8.27", height 11.69" Spacing 0.2". Whole paper must be with: Abstract: Time is a crucial factor in medical casualties. Emergency Medical Services (EMS) takes longer time leading to life loss, whether it's about an accident, out of hospital cardiac arrest (OOHCA) or drug delivery during the disaster. This paper proposes an efficient way to overcome this limitation by providing a medical aid aerially via a Multipurpose Medical Drone. A Quad-copter reaches the fatality spot employing GPS, carrying a payload containing components like AED (automatic external defibrillator), ECG, Temperature and Respiratory sensor to measure critical human body parameters. The measured values will be transmitted to a nearby hospital telemetrically using GSM or Wi-Fi for analyzing a patient's condition beforehand and for diagnosis. It can also be used to carry blood sample, critical drug depending on the situation. Drone aid can be got using the helpline or an app where location and the particularities describing the situation is entered and the aid is rendered to the required spot. This assistance can save lives preserving the precious seconds and reduces the death toll in casualties.
\end{abstract}

Key Words: EMS, GPS, Casualty Assistance, Defibrillator, ECG, Drug Delivery, Drone-aid app, IOT, OOHCA.

\section{INTRODUCTION}

Drones are flying robots that are technologically termed as Unmanned Aerial Vehicles (UAVs). UAV's have the capability to access locations that are difficult for humans to get to and are able to help in circumstances when time is crucial. Drones have a wide range of applications ranging from surveillance, search and rescue, delivery services and photography. Medical drones found to be the future for Medical Aids, still haven't stepped its feet in India. To overcome the limitations in conventional emergency ambulances, considerable efforts have been taken around the world- [3] fixed-wing drones have been developed in Rwanda. It forms an integral part of Rwanda's medical supply. A team of researchers recently tested whether a drone

Revised Manuscript Received on September 22, 2019.

Vaishali Nimilan (Student) ,Department Of Electronics and Instrumentation Engineering Easwari Engineering College (Autonomous Institution), Chennai 89. vaishalinimilan@gmail.com.

Dr.Gunaselvi Manohar (Professor), Department Of Electronics and Instrumentation Engineering Easwari Engineering College (Autonomous Institution), Chennai 89,gunaselvim@rediffmail.com.

R.Sudha (Assistant Professor),Department Of Electronics and Instrumentation Engineering Easwari Engineering College (Autonomous Institution), Chennai 89, sudharatnam@gmail.com.

Pearley Stanley (Assistant Professor), Department Of Electronics and Instrumentation Engineering Easwari Engineering College (Autonomous Institution), Chennai 89, pearleysolace@gmail.com.. or an ambulance had a faster response time in delivering an automated external defibrillator to a patient in cardiac arrest [2]. They compared the travel time of the drone with that of the emergency medical services. The researchers found that the drone arrived more quickly than EMS in all cases, with an average reduction in response time of about 16 minutes. During a medical emergency, those minutes can be the difference between life and death. Drones could carry defibrillators to heart attack victims faster than an ambulance. In the last ten years, road accidents have killed over 13 lakhs people in India. According to the reports from the Law Commission of India, 50\% of these were preventable injuries and the victims who died could have been saved if they had received proper care on time. The role of the bystander is critical in providing emergency care to the victim. But in India, bystanders are hesitant to help the injured due to the fear of legal procedures and repercussions. World Health Organization report claims that "skilled and empowered bystanders play a crucial role in saving lives" and in order to enable public to come forward and help injured persons, a supportive legal and ethical environment is needed and this environment was provided by the Supreme court of India on march 30, 2016 - Good Samaritan law stating that the eyewitness or the bystander who helps or informs the police will not be penalized bylaw.

\section{EXISTING SYSTEM}

The existing system is using only ambulance to help people in accidents and also going a step higher aerial solution has been proposed to deliver packages only during casualties or for surveillance or going farther an aerial solution has been proposed with distance constraints [4]. With the increasing population of vehicles, undisciplined drivers and poor infrastructure of roads has caused EMS wagon to often report late to the spot of casualty thereby reducing the chance of survival of the victim. The first one hour after the accident is said to be the golden hour to save the victim. If one can reach them within an hour and provide medical service, it can increase survival rate by $80 \%$. Unfortunately more $50 \%$ ambulance are said to report late to the spot after the golden hour and also many calls are being rejected due to lack of ambulance. .In rural areas where ambulance services are very limited the cases are even worse than cities. Another major issue is with the death due to unavailability of required blood 
group sample or required drug on time even if the patient is reported to hospital within an hour. In India, the roads are not ambulance friendly hence many patients with critical injuries are often declared 'brought dead'. This limitation has created void for further solutions which can consume less time and save lives. Further TU delft's initiative of delivering defibrillator for OOHCA has created new leap in redefining medical assistances [5].

\section{PROPOSED SYSTEM}

The solution proposed by us will use the time between the occurrence of an accident and arrival of an ambulance. EMS helpline will allow both ambulance and drone to reach casualty spot but the drone carrying aid will reach in fewer minutes in compared to ambulance which may take around half an hour to several hours to reach depending on the traffic. There is also a specialized application created which can be used to book a drone for emergency need by specifying the cause and location just like you book a taxi. Once the drone reaches, it will guide the public or the police personnel present there to assist the injured. Our project will be a handy tool for a person who is willing to help the injured during the scenario even if the person is or not from a medical background. The voice control module and the video module will give the person assistance on how to use the incorporated patient monitoring module consisting of respiratory, ECG and body temperature sensor and also inculpate him or her with some knowledge of what should how it should be fixed on the patient's body. The measured values of critical parameters are transmitted using IoT and GSM to the nearby hospital and ambulance, thereby preserving their time of diagnosing the patient only after the arrival of ambulance. The system not only proposes solution for accidents but also can redefine the way blood samples, antivenin or any other critical drug can be delivered to the place of need within minutes which also plays an important role in saving lives.

\section{*Refer Figure 1, 2}

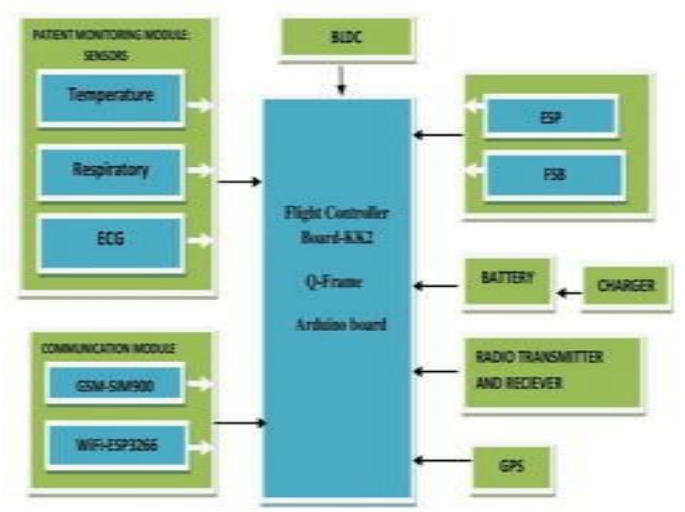

Figure 1: Block Diagram

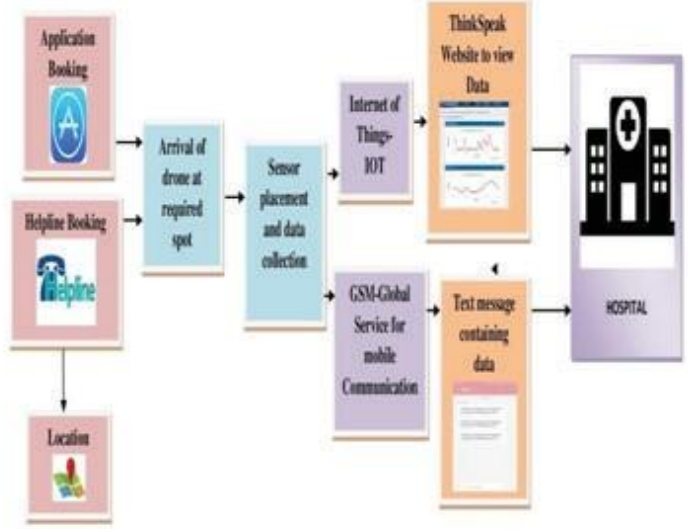

Figure 2: Methodological Approach

\section{METHODOLOGICAL APPROACH}

GPS allows the quad-copter to locate the spot and land itself. After landing it will assist the person present by using an audio and a video module attached to the quad-copter which will have a person in command to help the common man to attach the sensor to body of the injured. The Analog sensors start measuring the values and passes it to the Arduino board from there it is passed to the hospital and ambulance using GSM and Wi-Fi for analysis by a doctor or a person present in the ambulance. *Refer Figure 3

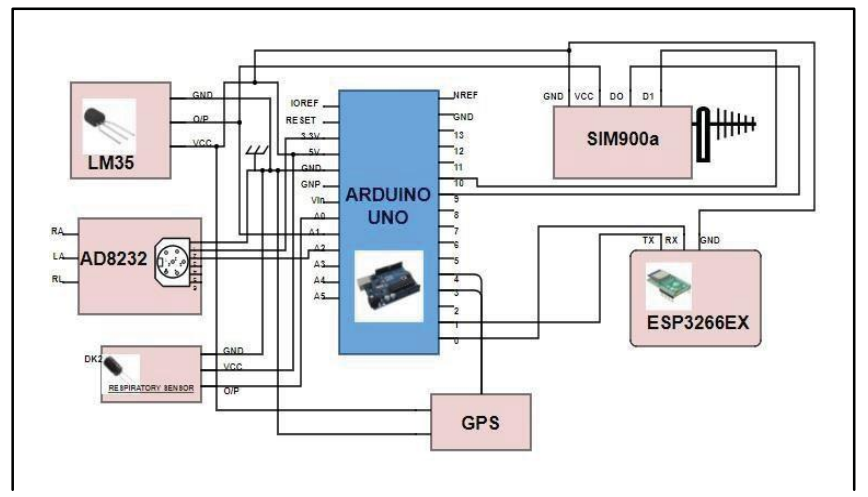

Figure 3: Schematic diagram

\section{COMPONENT DESCRIPTION}

There are two modules which consist of the drone components including frame and the patient monitoring module.

\section{A. DRONE FRAME: UAV frame:}

Every UAV needs a frame to house all the Components. They are chosen based upon the weight, size, and materials. Here an X-shaped frame is used to carry the FSB, ESC, Motor, Propellers and Receiver. 


\section{- Motors}

Motors are required to spin the Propellers. Motors are also chosen based upon the ESC it should be paired with. $\mathrm{RPM}=\mathrm{KV} * \mathrm{VOLTAGE} 1000 * 12=12000 \mathrm{RPM}$

\section{B. Electronic speed controllers (ESC8266):}

The ESC is what tells the motors how fast to spin at any given time. Every UAV needs four ESCs, one connected to each motor. The ESCs are then connected directly to FSB because the motors on a UAV must all spin at precise speeds to achieve accurate flight, so the ESC is very important.

\section{- Propellers}

An UAV has four propellers of which two are called "normal propellers" spinning counter-clockwise and the other two are called "pusher propellers" spinning clockwise to give balance during lift. The propeller size is 10 inches * 4.5 pitch.

\section{- Flight Stabilization Board}

FSB The flight control board is the 'brain' of the UAV. It houses various sensors including gyroscopes and accelerometers which determine the speed of UAV's motors spin. Board used is KK2. The four channels are AILERONS, ELEVATOR, THROTTLE, and RUDDER.

\section{- Radio transmitter and receiver}

The Uav is controlled in air by the transmission and receiving of the Radio Signal. The signal range is $2.4 \mathrm{GHz}$.

\section{- Li-Po battery}

UAVs typically use Li-Po batteries which come in a variety of sizes and configurations. This battery is rated at 12 volts. Li- Po batteries also have a power rating in $\mathrm{mAh}$ (which stands for milliamps per hour).A general rule of thumb is that doubling the battery power will get you $50 \%$ more flight time, assuming the UAV can lift the additional weight.

\section{- Arduino-Microcontroller Board}

The board used is Arduino Uno which is based on ATmega328P microcontroller. It has 14 digital input pins out of which 6 can be used for PWM output and 6 analog pins. It has operating range of $5 \mathrm{~V}$. It has $16 \mathrm{MHZ}$ quartz crystal, ICSP header and a reset button

\section{B. Patient Monitoring Module-Sensors AD8232-ECG}

The ECG sensor is used for measuring the heart's electrical activity which can be pictured as an Electrocardiogram and output as an Analog reading. AD8232 acts as an op amp obtaining a clear output signal from the PR-QT Intervals easily. The AD8232 is designed for obtaining, amplifying and filtering small bio-potential signals in the presence of external noise. There are nine connections from the IC to which pins, wires or other connectors can be soldered. SDN, LO+, LO-, OUTPUT, 3.3V, GND are the essential pins. RA (Right Arm), LA (Left Arm), and RL (Right Leg) pins are provided for attachment. An LED indicator light is provided for pulsating to the rhythm of a heart beat. *Refer Figure 4

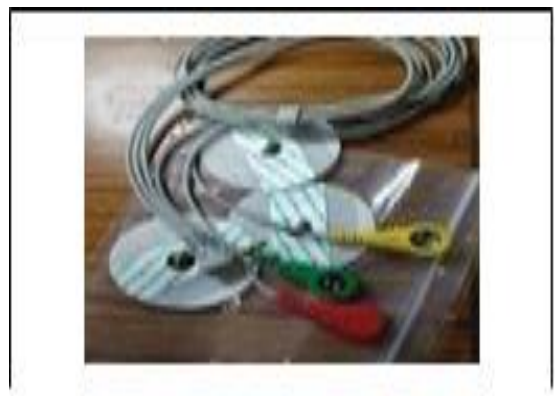

Figure 4: ECG Nodes

\section{A. Temperature Sensor-LM35}

The LM35 is one kind of commonly used temperature sensor that can be used to measure temperature with an electrical o/p comparative to the temperature (in ${ }^{\circ} \mathrm{C}$ ). It can measure temperature more correctly compare with a thermistor. This sensor generates a high output voltage than thermocouples and may not need that the output voltage is amplified. The LM35 has an output voltage that is proportional to the Celsius temperature. The scale factor is $.01 \mathrm{~V} /{ }^{\circ} \mathrm{C}$. The LM35 does not need any exterior calibration and maintains an exactness of $+/-0.4^{\circ} \mathrm{C}$ at room temperature and $+/-0.8^{\circ} \mathrm{C}$ over a range of $0^{\circ} \mathrm{C}$ to $100^{\circ} \mathrm{C}$. One more significant characteristic of this sensor is that it draws just 60 micro amps from its supply and acquires a low self-heating capacity.

\section{B. Respiratory sensor}

The respiratory rate of an adult is $12-20$ breaths per minute. When a person inhales or exhales the resistance of the chord changes which is proportional to the breathing rate of the individual. 


\section{RESULTS}

QUADCOPTER: FRAME:

Configuration: Cross " $\mathrm{X}$ "

Length: $41 \mathrm{~cm}$

Width: $41 \mathrm{~cm}$

Height:27 cm

\section{DESIRED FLIGHT CONDITION:}

Cruise velocity: $4 \mathrm{~m} / \mathrm{s}$

Altitude: $20 \mathrm{~m}$

Density: $1.05 \mathrm{~kg} / \mathrm{m} 3$

Viscosity: $17.87 * 10-6$

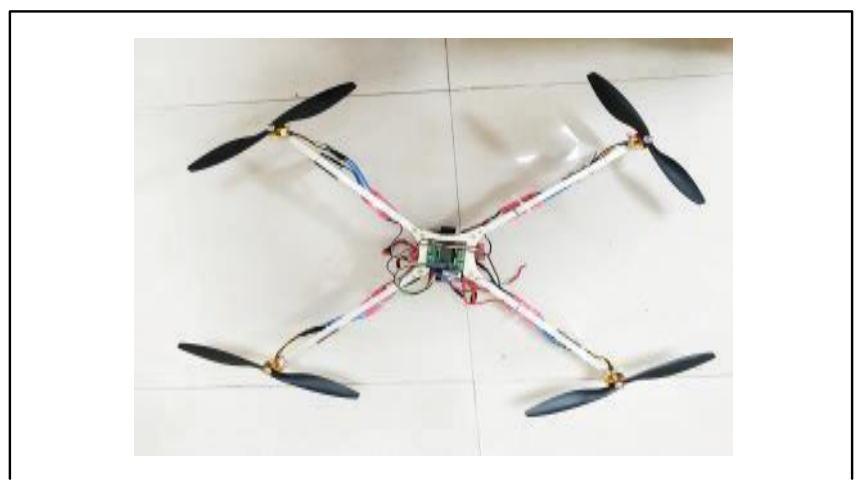

Figure 5: Quadcopter Topview

\section{A. THINGSPEAK- IOT} channel Id for every user. The sensor data are transmitted to the website every 20 seconds. Here the pictorial representation consists of three sensor outputs. The output will be transmitted using ESP3266EX. *Refer Figure 6

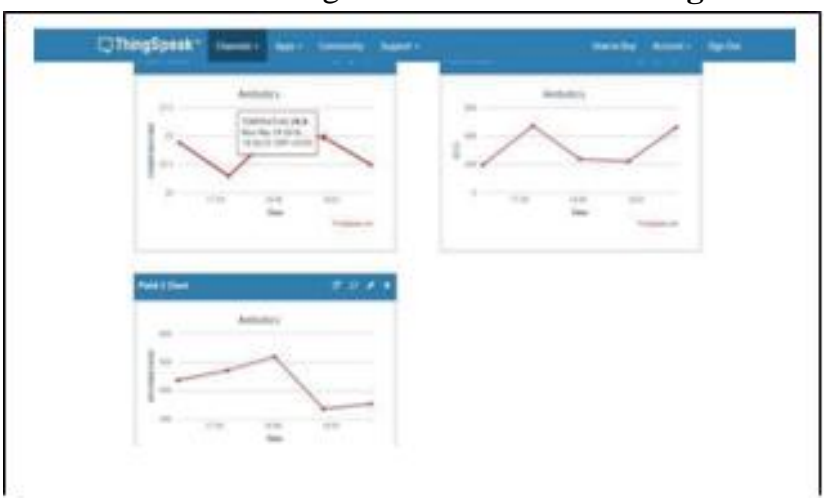

Figure 6: Iot Output

\section{B. FAIL PROOF-GSM:}

In case the internet connection fails to connect or work, the data is transmitted as a text message to the concerned person. The GSM module used is SIM900. So the personal will be able to see the patient's critical parameters through a text message.
The website provides a unique identification key and

\section{*Refer Figure 7}

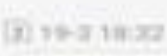

\section{Change in Temperature Temperatur e:42.97ECG:159Respiration-207}

\section{Change in Temperature Temperatur e:59.57ECG:153Respiration507}

\section{Change in Temperature Temperatur e:42.48ECG:402Respiration-207}

Figure 7: Text Message

\section{B. MED-DRONE APP}

An application Med-Drone is used to book the drone which gives an alternative and convenient method rather than reaching to the helpline. It will ask the person booking for the purpose, location and will intimate the arriving time of the drone as a message. *Refer Figure 8.

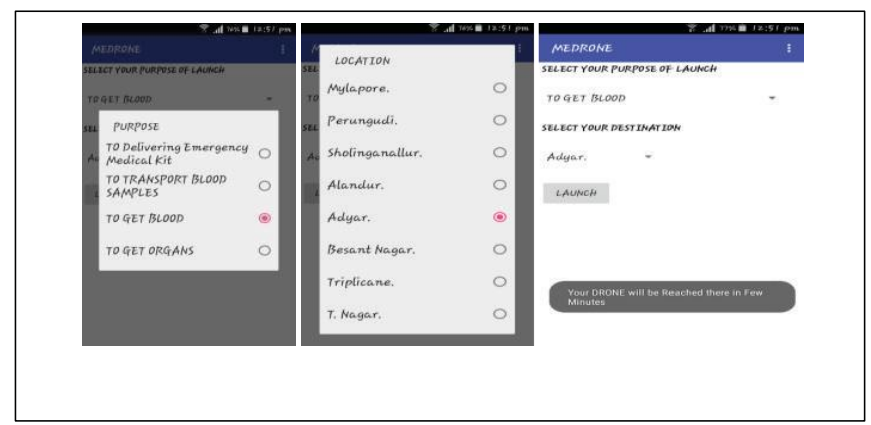

Figure 8: Med-Drone APP

\section{APPLICATIONS}

- Out of Hospital Cardiac arrest Assistance

- Causality Assistance

- Disaster time supplements

- Organ, Blood transmission

- Anti-venom, vaccines for rural areas

- Pharmaceutical drug delivery

\section{LIMITATIONS}

Drones suffer same problem as rockets-the more you lift, the more energy you need. Payload will be proportional to the design of the quad-copter. It is Difficult to deliver assistance during stormy or during heavy rain conditions. 


\section{CONCLUSION}

The project aims to save lives by providing assistance before the ambulance arrives. With the help of drone aid, the time elapsed during the arrival of ambulance can be saved. Effective administration of first aid can be carried out with the help of live screen interaction with doctors and quicker determination of critical body parameters and transmit it to the nearby hospital using Iot in order to make prior surgical arrangements which in turn increase the probability of saving lives. Aerial medical assistance also includes delivering medical drugs and blood samples during emergencies.

\section{REFERENCES}

1) Cornelius A Thiels, Johnathon M Aho, Scott P Zietlow, Donald H Jenkins "Use of unmanned aerial vehicles for medical product transport", Scandinavian Journal of Trauma,2016

2) A Claesson, D Fredman, L Svenson, M Ringh "Unmaanned aerial vehicle (drones) in out-of-hospital - cardiac -arrest, Air medical Journal,2015

3) Medical delivery Drones take flight in East Africa- Zipline , IEEE Spectrum( Volume 55, Issue:1, January 2018)

4) A.Josephin Arockia Dhivya, "Quadcopter based technology for an emergency healthcare “ Biosignals,Images, andInstrumentation(ICBSII) Conference, 2017, 17291128

5) TU delft ,"Ambulance drone", Alec momont,2014 https://www.tudelft.nl/en/2014/tu-delft/tu-delftsambulance-drone-drastically-increases-chances-of- survival-of-cardiacpatients/arrest- 